\title{
Fixed Point Formulation Using Exponential Logarithmic Transformations and Its Applications
}

\author{
C. Ganesa Moorthy \\ Department of Mathematics, Alagappa University, Karaikudi-630 003, India
}

\author{
Article Info \\ Keywords: Complete metric space, \\ Compact metric space, Hausdorff met- \\ ric \\ 2010 AMS: $54 H 25,37 C 25$ \\ Received: 14 August 2020 \\ Accepted: 04 November 2020 \\ Available online: 15 December 2020
}

\begin{abstract}
The axioms for a metric $D$ were transformed into axioms of the function $\exp D$, and a new generalized metric called multiplicative metric was introduced in 2008 based on these transformed axioms. A review of a method of converting metric fixed point results through logarithmic transformation to multiplicative metric fixed point results and converting multiplicative metric fixed point results through exponential transformation to metric fixed point results has been presented. Applications of this procedure have also been discussed.
\end{abstract}

\section{Introduction}

Fixed point theory accommodates many types of distance functions. On many occasions distance functions are introduced just to derive fixed point theorems and this is justified in the survey articles ([1],[2]). The survey article [2] presents conversions of metric fixed point results through exponential logarithmic transformations into fixed point results on a class of generalized metric spaces which are called multiplicative metric spaces. The concept of a multiplicative metric was just mentioned in the article [3] in the year 2008. But, other researchers used this concept to derive fixed point results by transforming arguments of proofs of results of metric fixed point theory. The survey article [2] applied transformations on statements instead of proofs, by referring to many research articles. The present article is to review these techniques of applying transformations, but by referring to books having collections of results with proofs. It is concluded as in [2] that there is a one to one correspondence between metric fixed point results and multiplicative metric fixed point results. Exact applications are also mentioned. The main purpose of applying transformations is to obtain many examples and to obtain new results. The main purpose of fixed point theory is to solve equations.

Only very few results are selected, mostly from the book [4], for transformation, and new articles like [5] are not considered for transformations. The statements of these results chosen for transformations are not presented for management of symbols. One more thing should be mentioned. The name "multiplicative metric" given in [3] is changed into "EL metric" to stress the usefulness of transformations. The usual notation used for a multiplicative metric is also changed in this article for convenience. It should be mentioned that apart from special nonlinear transformations there are special linear transformations. See for example [6]-[9].

\section{Transformed metrics and topologies}

Let us first recall the definition of a metric $D$. 
Definition 2.1. [1] Let $X$ be a non empty set. Let $D$ be a real valued function on $X \times X$ satisfying the following axioms.

(I) $D(x, y) \geq 0$, for all $x, y \in X$

(II) $D(x, y)=D(y, x)$, for all $x, y \in X$

(III) $D(x, y) \leq D(x, z)+D(z, y)$, for all $x, y, z \in X$

(IV) $D(x, y)=0$ if and only if $x=y$ in $X$.

Then $D$ is called a metric on $X$. The pair $(X, D)$ is called a metric space.

Definition 2.2. [3] Let $X$ be a non empty set. Let $d$ be a real valued function on $X \times X$ satisfying the following axioms.

(i) $d(x, y) \geq 1$, for all $x, y \in X$

(ii) $d(x, y)=d(y, x)$, for all $x, y \in X$

(iii) $d(x, y) \leq d(x, z) d(z, y)$, for all $x, y, z \in X$

(iv) $d(x, y)=1$ if and only if $x=y$ in $X$.

Then $d$ is called an EL metric on $X$. The pair $(X, d)$ is called an EL metric space.

E and L are used for the words "Exponential" and "Logarithmic". So, EL metrics are our transformed metrics. The word "generalized" is suppressed in giving name in Definition 2.2, because metrics are to be derived from EL metrics and EL metrics are to be derived from metrics.

Let $(X, D)$ be a metric space. Let us use the notation “ $\exp D$ ” for the composite function which is defined by

$$
(\exp D)(x, y)=\exp (D(x, y))=e^{D(x, y)}, \text { for all } x, y \in X .
$$

Let $d=\exp D$. Then $d$ satisfies the conditions (i),(ii),(iii) and (iv) given in Definition 2.2. Thus, $(X, d)$ is an EL metric space, when $d=\exp D$.

On the other hand, let us consider an EL metric space $(X, d)$. Let us use the notation "log $d$ " for the composite function which is defined by

$$
(\log d)(x, y)=\log (d(x, y)), \text { for all } x, y \in X .
$$

Let $D=\log d$. Then $D$ satisfies the conditions (I),(II),(III) and (IV) given in Definition 2.1. Thus $(X, D)$ is a metric space, when $D=\log d$.

These two transformations provide fundamental techniques required to convert classical fixed point results for metric spaces into fixed point results for EL metric spaces. Let us first introduce technical terms required for transformations in results. Let us always associate $D$ for a metric and $d$ for an EL metric, even if it is not mentioned explicitly. Some times, the variations like $D_{X}, D_{Y}, D_{1}, D_{2}$, and $d_{X}, d_{Y}, d_{1}, d_{2}$ will be used for these purposes. Let us assume all classical definitions for open sets, closed sets, closures, topology induced by a metric, Cauchy sequences, convergent sequences, complete metric spaces, totally bounded metric spaces, compact spaces, etc., corresponding to metric spaces.

Notation 2.3. Let $(X, D)$ be a metric space. For each $r \geq 0$, and for each $x \in X$, let $B_{D}(x, r)=\{y \in X: D(x, y)<r\}$ and $C_{D}(x, r)=\{y \in X: D(x, y) \leq r\}$ denote open balls and closed balls.

Definition 2.4. [10] Let $(X, d)$ be an EL metric space. For each $r \geq 1$, and for each $x \in X$, let $B_{d}(x, r)=\{y \in X: d(x, y)<r\}$ and $C_{d}(x, r)=\{y \in X: d(x, y) \leq r\}$. Let us call them also as open ball and closed ball having centre $x$ and radius $r$. Let $u s$ say that $(X, d)$ is totally bounded if for every $r>1, X$ is covered by finitely many open balls with radius $r$.

Remark 2.5. [10] Let $d$ be an EL metric on $X$. Let $D=\log d$ so that $d=\exp D$. Then $B_{D}(x, r)=B_{d}(x, \exp r)$, because $D(x, y)<r$ if and only if $d(x, y)<\exp r$, for $r>0$. Similarly, $C_{D}(x, r)=C_{d}(x, \exp r)$, for $r>0$. So, $(X, d)$ is totally bounded if and only if $(X, D)$ is totally bounded.

Definition 2.6. [10] A subset A of an EL metric space $(X, d)$ is said to be open, iffor each $x \in A$, there is a number $r>1$ such that $B_{d}(x, r) \subseteq A$. Let $\tau_{d}=\{A \subseteq X: A$ is open in $(X, d)\}$.

Remark 2.7. [10] Let $A$ be a subset of an EL metric space $(X, d)$. Then $A \in \tau_{d}$ if and only if it is open with respect to the topology $\tau_{D}$ induced by $D=\log d$. This is a consequence of Remark 2.5. Thus $\tau_{d}$ is a topology and it coincides with $\tau_{D}$. Let $u s$ say that $(X, d)$ is compact, if $\left(X, \tau_{d}\right)$ is compact.

Definition 2.8. [10] Let $\left(x_{n}\right)_{n=1}^{+\infty}$ be a sequence in an EL metric space $(X, d)$. Then $\left(x_{n}\right)_{n=1}^{+\infty}$ is said to be Cauchy in $(X, d)$, if for every $\varepsilon>1$, there is an integer $n_{0}$ such that $d\left(x_{n}, x_{m}\right)<\varepsilon$, for all $n, m \geq n_{0}$. The sequence $\left(x_{n}\right)_{n=1}^{+\infty}$ is said to be convergent in $(X, d)$, if for every $\varepsilon>1$, there is an element $x \in X$, and there is an integer $n_{0}$ such that $d\left(x_{n}, x\right)<\varepsilon$, for all $n \geq n_{0}$. Let $u$ s say that $\left(x_{n}\right)_{n=1}^{+\infty}$ converges to a limit point $x$ in $(X, d)$, in this case. An EL metric space is said to be complete, if every Cauchy sequence is convergent in it. 
Remark 2.9. [10] Let $\left(x_{n}\right)_{n=1}^{+\infty}$ be a sequence in an EL metric space $(X, d)$. Let $D=\log d$. By Remark 2.5 , $\left(x_{n}\right)_{n=1}^{+\infty}$ is Cauchy in $(X, d)$ if and only if it is Cauchy in $(X, D)$. Also, $\left(x_{n}\right)_{n=1}^{+\infty}$ is convergent to $x$ in $(X, d)$ if and only if it is convergent to $x$ in $(X, D)$. In particular, every convergent sequence in $(X, d)$ is Cauchy. Moreover, $(X, d)$ is complete if and only if $(X, D)$ is complete.

A transformation method can be applied for arguments of proofs. The first illustration for this action is a proof of the following known fact.

Proposition 2.10. Let $(X, d)$ be an EL metric space. Let $\left(x_{n}\right)_{n=1}^{+\infty}$ be a sequence converging to $x$ in $(X, d)$. Then $\left(x_{n}\right)_{n=1}^{+\infty}$ is Cauchy in $(X, d)$.

Proof. Fix $\varepsilon>1$. Then there is an integer $n_{0}$ such that $d\left(x_{n}, x\right)<\sqrt{\varepsilon}$, for all $n \geq n_{0}$. Then $d\left(x_{n}, x_{m}\right) \leq d\left(x_{n}, x\right) d\left(x, x_{m}\right)<\varepsilon$, for all $n, m \geq n_{0}$. This completes the proof.

Let us establish two more results which can also be obtained by applying transformation directly to the known results.

Lemma 2.11. Let $(X, d)$ be an EL metric space. Then

$$
1 \leq \max \left\{\frac{d(u, v)}{d(x, y)}, \frac{d(x, y)}{d(u, v)}\right\} \leq d(u, x) d(v, y), \text { for all } x, y, u, v \in X
$$

Proof. $d(u, v) \leq d(u, x) d(x, y) d(y, v)$. So, $\frac{d(u, v)}{d(x, y)} \leq d(u, x) d(y, v)$. Similarly, $\frac{d(x, y)}{d(u, v)} \leq d(u, x) d(y, v)$. Now, the lemma follows.

Proposition 2.12. Let $(X, d)$ be an EL metric space. Let $\left(x_{n}\right)_{n=1}^{+\infty}$ converge to $x$ and $\left(y_{n}\right)_{n=1}^{+\infty}$ converge to $y$ in $(X, d)$. Then $d\left(x_{n}, y_{n}\right) \rightarrow d(x, y)$ as $n \rightarrow+\infty$.

Proof.

$$
1 \leq \max \left\{\frac{d\left(x_{n}, y_{n}\right)}{d(x, y)}, \frac{d(x, y)}{d\left(x_{n}, y_{n}\right)}\right\} \leq d\left(x_{n}, x\right) d\left(y_{n}, y\right), \text { for all } n,
$$

by Lemma 2.11. The result follows, because $d\left(x_{n}, x\right) \rightarrow 1$ and $d\left(y_{n}, y\right) \rightarrow 1$ as $n \rightarrow+\infty$.

Let us now transform arguments of the proof of the metrization lemma ([11], Chapter 6, Lemma 12).

Lemma 2.13. Let $\left(U_{n}\right)_{n=0}^{+\infty}$ be a sequence of symmetric subsets of $X \times X$ such that $U_{0}=X \times X$, each $U_{n}$ contains the diagonal $\Delta=\{(x, x): x \in X\}, U_{n+1} \circ U_{n+1} \circ U_{n+1} \subseteq U_{n}$ for each $n$, and such that $\Delta=\bigcap_{n=1}^{+\infty} U_{n}$. Then there is an EL metric $d$ on $X$ (that is, a function on $X \times X)$ such that $U_{n} \subseteq\left\{(x, y) \in X \times X: d(x, y)<5^{2^{-n}}\right\} \subseteq U_{n-1}$, for each positive integer $n$.

Proof. Define a function $f: X \times X \rightarrow[1,+\infty)$ by $f(x, y)=5^{2^{-n}}$ if and only if $(x, y) \in U_{n-1} \backslash U_{n}$ and by $f(x, x)=1$, for all $x \in X$. For every $x, y \in X$, let $d(x, y)=\inf \prod_{i=0}^{n} f\left(x_{i}, x_{i+1}\right)$, where infimum is taken over all finite sequences (or chains) $x_{0}, x_{1}, \ldots, x_{n+1}$ such that $x=x_{0}$ and $y=x_{n+1}$. Then $d(x, y) \geq 1$ and $d(x, y) \leq d(x, z) d(z, y)$, for all $x, y, z \in X$. Also, $f(x, y)=f(y, x)$, for all $x, y \in X$, because each $U_{n}$ is symmetric. So, $d(x, y)=d(y, x)$, for all $x, y \in X$. Moreover, $d(x, x)=1$, for all $x \in X$, because $f(x, x)=1$, for all $x \in X$. Since $d(x, y)<5^{2^{-n}}$, for all $(x, y) \in U_{n}$, then $U_{n} \subseteq\left\{(x, y) \in X \times X: d(x, y)<5^{2^{-n}}\right\}$. Let us claim that, for any chain $x_{0}, x_{1}, \ldots, x_{n+1}, f\left(x_{0}, x_{n+1}\right) \leq\left(\prod_{i=0}^{n} f\left(x_{i}, x_{i+1}\right)\right)^{2}$. Let us establish the claim by induction on $n$. Let us call $n$ as the length of the chain $x_{0}, x_{1}, \ldots, x_{n+1}$. The claim is true for any chain with length $n=0$, because $f(x, y) \geq 1$, for all $x, y \in X$. Let us assume for induction that the claim is true for any chain with length less than $n$, and $n \geq 1$. To complete the proof of our claim, let us consider a chain $x_{0}, x_{1}, \ldots, x_{n+1}$ in $X$ such that $f\left(x_{i}, x_{i+1}\right)>1$, for all $i$. Let $a=\prod_{i=0}^{n} f\left(x_{i}, x_{i+1}\right)$. Let $k$ be the largest integer such that $\prod_{i=0}^{k} f\left(x_{i}, x_{i+1}\right) \leq \sqrt{a}$. Then $\prod_{i=k+1}^{n} f\left(x_{i}, x_{i+1}\right) \leq \sqrt{a}$. Hence, by our induction hypothesis, $f\left(x_{0}, x_{k}\right) \leq a$ and $f\left(x_{k+1}, x_{n+1}\right) \leq a$. Moreover, $f\left(x_{k}, x_{k+1}\right) \leq a$. If $m$ is the smallest integer such that $5^{2^{-m-1}} \leq a$, then $\left(x_{0}, x_{k}\right),\left(x_{k}, x_{k+1}\right),\left(x_{k+1}, x_{m+1}\right) \in U_{m}$ and hence $\left(x_{0}, x_{n+1}\right) \in U_{m-1}$. Hence $f\left(x_{0}, x_{n+1}\right) \leq 5^{2^{-m}} \leq a^{2}$. Thus our claim is true. This claim proves that $\left\{(x, y) \in X \times X: d(x, y)<5^{2^{-n}}\right\} \subseteq U_{n-1}$, for all $n=1,2, \ldots$. This completes the proof. 
One should compare the previous proof with the proof of ([11], Chapter 6, Lemma 12). This comparison is necessary to understand the technique of applying transformation in arguments of proofs. Note that the exponential function does not appear in the previous proof. This one happens, because $5^{d(x, y)}$ is also a metric, when $\mathrm{d}$ is an EL metric. One can also observe that the statement of ([11], Chapter 6, Lemma 12) can be directly converted into the statement of Lemma 2.13 of this article without going through proofs. Lemma 2.13 of this article and ([11], Chapter 6, Lemma 12) jointly imply the following. The topology induced by an EL metric is also induced by a metric, and the topology induced by a metric is also induced by an EL metric. But, this was already implied by Remark 2.7 .

Conclusion 2.14. If $d$ is an EL metric and $D=\log d$, then topologies, convergent sequences, Cauchy sequences, continuity, compactness, and completeness are common for both $d$ and D. Both $d$ and D are jointly continuous. All these fundamental facts will be applied in this article.

\section{Advantage of transformations in arguments}

Theorem 3.1. Let $(X, d)$ be a compact EL metric space. Let $T: X \rightarrow X$ be a mapping such that $d\left(T^{2} x, T x\right)<d(T x, x)$, whenever $T x \neq x$ in $X$. Then $T$ has a fixed point. Moreover, if $d(T x . T y)<d(x, y)$, whenever $x \neq y$ in $X$, then $T$ has a unique fixed point $x^{*}$, and $\left(T^{n} x_{0}\right)_{n=1}^{+\infty}$ converges to $x^{*}$, for any fixed $x_{0}$ in $X$.

Proof. Let $\phi(x)=d(T x, x)$, for all $x \in X$. Then $\phi$ has a minimum at some $x^{*} \in X$. If $T x^{*} \neq x^{*}$, then

$$
1 \leq \phi\left(T x^{*}\right)=d\left(T^{2} x^{*}, T x^{*}\right)<d\left(T x^{*}, x^{*}\right)=\phi\left(x^{*}\right) .
$$

So, $T x^{*}=x^{*}$, because $\phi$ has a minimum at $x^{*}$.

Suppose further that $d(T x, T y)<d(x, y)$, whenever $x \neq y$ in $X$. Suppose $T x^{* *}=x^{* *}$ and $T x^{*}=x^{*}$ for two points $x^{*}, x^{* *}$ in $X$. If $x^{*} \neq x^{* *}$, then

$$
1 \leq d\left(x^{* *}, x^{*}\right)=d\left(T x^{* *}, T x^{*}\right)<d\left(x^{* *}, x^{*}\right)
$$

So, $x^{*}=x^{* *}$. Thus $T$ has a unique fixed point $x^{*}$ in $X$. Fix $x_{0}$ in $X$. Let $c_{n}=d\left(T^{n} x_{0}, x^{*}\right)$. If $c_{m}=1$ for some $m$, then $\left(T^{n} x_{0}\right)_{n=1}^{+\infty}$ converges to $x^{*}$ in $(X, d)$. Suppose $c_{n} \neq 1$ for every $n$. Then

$$
1 \leq c_{n+1} \leq d\left(T^{n+1} x_{0}, x^{*}\right)=d\left(T\left(T^{n} x_{0}\right), T x^{*}\right)<d\left(T^{n} x_{0}, x^{*}\right)=c_{n}, \text { for all } n .
$$

Let $\lim _{n \rightarrow+\infty} c_{n}=c$. If $c=1$, then $\left(T^{n} x_{0}\right)_{n=1}^{+\infty}$ converges to $x^{*}$ in $(X, d)$. Suppose $c>1$. Let $\left(T^{n_{i}} x_{0}\right)_{i=1}^{+\infty}$ be a subsequence of $\left(T^{n} x_{0}\right)_{n=1}^{+\infty}$ such that the subsequence converges to some $z$ in $(X, d)$. Then

$$
1<c=\lim _{i \rightarrow+\infty} c_{n_{i}}=\lim _{i \rightarrow+\infty} d\left(T^{n_{i}} x_{0}, x^{*}\right)=d\left(z, x^{*}\right)
$$

so that $x^{*} \neq z$. Since $T$ is continuous,

$$
1<c=\lim _{i \rightarrow+\infty} d\left(T^{n_{i}+1} x_{0}, x^{*}\right)=d\left(T z, x^{*}\right)=d\left(T z, T x^{*}\right)<d\left(z, x^{*}\right)=c
$$

This is impossible. Thus $c=1$, and the result follows.

Corollary 3.2. Let $(X, D)$ be a compact metric space. Let $T:(X, D) \rightarrow(X, D)$ be a mapping such that $D(T x, T y)<D(x, y)$, for $x \neq y$ in $X$. Then there is a unique fixed point $x^{*}$ in $X$. Moreover, for any $x_{0} \in X,\left(T^{n} x_{0}\right)_{n=1}^{+\infty}$ converges to $x^{*}$ in $(X, D)$.

Proof. Define $d=\exp D$. Then $(X, d)$ is a compact EL metric space. Also, $d(T x, T y)<d(x, y)$, for $x \neq y$ in $X$. So, the result follows from Theorem 3.1.

The previous Corollary 3.2 is ([4], Theorem 3.5). The following Corollary 3.4 is the most fundamental theorem of metric fixed point theory. It is ([4], Theorem 3.1), which is the Banach contraction principle.

Theorem 3.3. Let $(X, d)$ be a complete EL metric space. Let $k \in(0,1)$. Let $T: X \rightarrow X$ be a mapping such that $d(T x, T y) \leq$ $(d(x, y))^{k}$, for all $x, y \in X$. Then $T$ has a unique fixed point $x^{*}$. Also, for each $x_{0}$ in $X$, the sequence $\left(T^{n} x_{0}\right)_{n=1}^{+\infty}$ converges to $x^{*}$ in $(X, d)$. Moreover,

$$
d\left(T^{n} x_{0}, x^{*}\right) \leq\left(d\left(x_{0}, T x_{0}\right)\right)^{\frac{k^{n}}{1-k}}, \text { for all } n
$$


Proof. Fix $x_{0} \in X$. Let $x_{n}=T^{n} x_{0}$, for all $n=1,2,3 \ldots$. Then $d\left(x_{n+1}, x_{n}\right) \leq\left(d\left(x_{n}, x_{n-1}\right)\right)^{k} \leq\left(d\left(x_{1}, x_{0}\right)\right)^{k^{n}}$. For $m>n$,

$$
\begin{aligned}
1 & \leq d\left(x_{n}, x_{m}\right) \\
& \leq d\left(x_{n}, x_{n+1}\right) d\left(x_{n+1}, x_{n+2}\right) \ldots d\left(x_{m-1}, x_{m}\right) \\
& \leq\left(d\left(x_{1}, x_{0}\right)\right)^{k^{n}+k^{n+1}+\ldots+k^{m-1}} \\
& \leq\left(d\left(x_{1}, x_{0}\right)\right)^{k^{n}+k^{n+1}+\ldots} \\
& =\left(d\left(x_{1}, x_{0}\right)\right)^{\frac{k^{n}}{1-k}}
\end{aligned}
$$

Since the right hand side tends to 1 as $n \rightarrow+\infty,\left(x_{n}\right)_{n=1}^{+\infty}$ is a Cauchy sequence that converges to some element $x^{*}$ in $(X, d)$. Moreover, when $\mathrm{m}$ tends to infinity in the previous relation, it is obtained that

$$
1 \leq d\left(x_{n}, x^{*}\right) \leq\left(d\left(T x_{0}, x_{0}\right)\right)^{\frac{k^{n}}{1-k}}
$$

for every $n=1,2, \ldots$. If $n$ tends to infinity in this relation, then it is concluded that $d\left(x_{n}, x^{*}\right) \rightarrow 1$ as $n \rightarrow+\infty$. Since

$$
1 \leq d\left(T x^{*}, x^{*}\right)=\lim _{n \rightarrow+\infty} d\left(x_{n+1}, x_{n}\right) \leq \lim _{n \rightarrow+\infty}\left(d\left(x_{1}, x_{0}\right)\right)^{k^{n}}=1
$$

it follows that $T x^{*}=x^{*}$. Moreover, if $T x^{* *}=x^{* *}$, then

$$
1 \leq d\left(x^{*}, x^{* *}\right)=d\left(T x^{*}, T x^{* *}\right) \leq\left(d\left(x^{*}, x^{* *}\right)\right)^{k} .
$$

Since $k \in(0,1)$, it follows that $x^{*}=x^{* *}$. That is, $T$ has a unique fixed point $x^{*}$ in $X$.

Corollary 3.4. Let $(X, D)$ be a complete metric space. Let $k \in(0,1)$. Let $T: X \rightarrow X$ be a mapping such that $D(T x, T y) \leq$ $k D(x, y)$, for all $x, y \in X$. Then $T$ has a unique fixed point $x^{*}$. Moreover, for any $x_{0}$ in $X$, the sequence $\left(T^{n} x_{0}\right)_{n=1}^{+\infty}$ converges to $x^{*}$.

Proof. Let $d=\exp D$. Then $(X, d)$ is a complete EL metric space. Also, $d(T x, T y) \leq(d(x, y))^{k}$, for all $x, y \in X$. Corollary 3.4 now follows from Theorem 3.3.

Conclusion 3.5. One may understand that the proofs of Theorem 3.1 and Theorem 3.3 use arguments which resemble classical arguments and the arguments used in this article are obtained from classical arguments by means of logarithmic transformation. These types of transformed arguments should be developed, because one can derive fixed point results for metric spaces, as it is illustrated in Corollary 3.2 and Corollary 3.4.

\section{Transformation in statements}

Sometimes, it would be convenient to establish existence of fixed points in particular examples, when transformed statements of results are applied. This is the main advantage of transformation of statements of results.

Example 4.1. Let $X=\left[\frac{1}{2}, 2\right]$. Let $d(x, y)=\max \left\{x y^{-1}, y x^{-1}\right\}$, for all $x, y \in X$. Then $(X, d)$ is a compact EL metric space. Define $T: X \rightarrow X$ by $T x=x^{\frac{1}{2}}$, for all $x \in X$. Then $d(T x, T y)<d(x, y)$ whenever $x \neq y$. This mapping $T$ has a unique fixed point 1 . For any fixed $x \in X, T^{n} x \rightarrow 1$ as $n \rightarrow+\infty$.

One may understand that the following Corollary 4.2 can be associated with Example 4.1. Although Corollary 4.2 is a part of Theorem 3.1, let us derive it from Corollary 3.2 for an illustration of transformation techniques.

Corollary 4.2. Let $(X, d)$ be a compact EL metric space. Let $T: X \rightarrow X$ be a mapping such that $d(T x, T y)<d(x, y)$, for all $x, y \in X$. Then $T$ has a unique fixed point $x^{*}$. Moreover, for any $x_{0}$ in $X,\left(T^{n} x_{0}\right)_{n=1}^{+\infty}$ converges to $x^{*}$ in $(X, d)$.

Proof. Let $D=\log d$. Then $(X, D)$ is a compact metric space, and $D(T x, T y)<D(x, y)$, whenever $x \neq y$ in $X$. Now, Corollary 3.2 implies Corollary 4.2.

Corollary 4.3. Let $(X, d)$ be a complete EL metric space. Let $k \in(0,1)$. Let $T: X \rightarrow X$ be a mapping such that $d(T x, T y) \leq$ $(d(x, y))^{k}$, for all $x, y \in X$. Then $T$ has a unique fixed point.

Proof. Let $D=\log d$. Then $(X, D)$ is a complete metric space and $D(T x, T y) \leq k D(x, y)$, for all $x, y \in X$. Now, Corollary 3.4 implies Corollary 4.3. 
Corollaries 3.2, 3.4, 4.2 and 4.3 are evidences for existence of a one to one correspondence between fixed point results for metric spaces and fixed point results for EL metric spaces. Since fixed point results for metric spaces have already been established, let us go for transformations of fixed point results for metric spaces. Let us consider some results from the book [4] for transformation.

Theorem 4.4. Let $(X, d)$ be a complete EL metric space. Let $k \in(0,1)$. Let $T: X \rightarrow X$ be a mapping such that $d(T x, T y) \leq$ $(d(x, y))^{k}$, for all $x, y \in X$. Let $x^{*}$ be the unique fixed point of $T$. Let $\left(\varepsilon_{n}\right)_{n=1}^{+\infty}$ be a sequence of numbers such that $\lim _{n \rightarrow+\infty} \varepsilon_{n}=1$ and $\varepsilon_{n}>1$, for all $n$. Let $\left(y_{n}\right)_{n=1}^{+\infty}$ be a sequence in $X$ such that $d\left(y_{n+1}, T y_{n}\right) \leq \varepsilon_{n}$, for all $n=1,2, \ldots$ Then $\lim _{n \rightarrow+\infty} y_{n}=x^{*}$.

Proof. Let $D=\log d$. Then $D(T x, T y) \leq k D(x, y)$, for all $x, y \in X$. Also, $\left(\log \varepsilon_{n}\right)_{n=1}^{+\infty}$ is a sequence of positive numbers such that $D\left(y_{n+1}, T y_{n}\right) \leq \log \varepsilon_{n}$, for all $n=1,2, \ldots$, and such that $\log \varepsilon_{n} \rightarrow 0$ as $n \rightarrow+\infty$. Now, ([4], Theorem 3.2) implies the result.

Theorem 4.5. Let $(X, d)$ be a complete EL metric space. Let $k \in(0,1)$. Let $n$ be a positive integer. Let $T: X \rightarrow X$ be a mapping such that $d\left(T^{n} x, T^{n} y\right) \leq(d(x, y))^{k}$, for all $x, y \in X$. Then $T$ has a unique fixed point.

Proof. Let $D=\log d$. Then $D\left(T^{n} x, T^{n} y\right) \leq k D(x, y)$, for all $x, y \in X$. By ([4], Theorem 3.3), the result follows.

Theorem 4.6. Let $(X, d)$ be a complete EL metric space. Let $\alpha:[1,+\infty) \rightarrow[0,1)$ be a function which satisfies the condition “ $\alpha\left(t_{n}\right) \rightarrow 1$ implies $t_{n} \rightarrow 1$ ”. Let $T: X \rightarrow X$ be a mapping such that $d(T x, T y) \leq(d(x, y))^{\alpha(d(x, y))}$, for all $x, y \in X$. Then $T$ has $a$ unique fixed point $x^{*}$. Also, for each $x_{0}$ in $X,\left(T^{n} x_{0}\right)_{n=1}^{+\infty}$ converges to $x^{*}$.

Proof. Let $D=\log d$. Let $A:[0,+\infty) \rightarrow[0,1)$ be a mapping defined by $A(t)=\alpha(\exp t)$, for all $t \in(0,+\infty)$. If $A\left(t_{n}\right) \rightarrow 1$, then $\alpha\left(\exp t_{n}\right) \rightarrow 1, \exp t_{n} \rightarrow 1$, and $t_{n} \rightarrow 0$ as $n \rightarrow+\infty$. Moreover,

$$
\begin{aligned}
D(T x, T y) & \leq \alpha(d(x, y)) D(x, y) \\
& =\alpha(\exp \log d(x, y)) D(x, y) \\
& =A(D(x, y)) D(x, y), \text { for all } x, y \in X .
\end{aligned}
$$

The conditions required for ([4], Theorem 3.6) are satisfied for $T:(X, D) \rightarrow(X, D)$ in the complete metric space $(X, D)$. Now, ([4], Theorem 3.6) implies the result.

Definition 4.7. Let $(X, d)$ be an EL metric space. For a subset $A$ of $X$, diameter of $A$ is denoted by diam $A$ and defined by $\operatorname{diam} A=\sup \{d(x, y): x, y \in A\}$. The subset $A$ is said to be a bounded set, if diam $A$ is finite. The EL metric space $(X, d)$ is bounded, if diam $X$ is finite.

Let $D=\log d$ for an EL metric $d$ on $X$. A subset $A$ of $X$ is bounded in the metric space $(X, D)$ if and only if it is bounded in the EL metric space $(X, d)$.

Theorem 4.8. Let $(X, d)$ be a bounded EL metric space. Let $\psi:[1,+\infty) \rightarrow[1,+\infty)$ be a function such that $\psi$ is continuous from right at all points and such that $1<\psi(r)<r$ for $r>1$. Let $T:(X, d) \rightarrow(X, d)$ be a continuous mapping, with respect to the topology induced by $d$, such that $d(T x, T y) \leq \psi(d(x, y))$, for all $x, y \in X$. Then $T$ has a unique fixed point $x^{*}$. Also, for each $x_{0}$ in $X,\left(T^{n} x_{0}\right)_{n=1}^{+\infty}$ converges to $x^{*}$ as $n \rightarrow+\infty$.

Proof. Let $D=\log d$ so that $(X, D)$ is a complete metric space and $T:(X, D) \rightarrow(X, D)$ is continuous. Define $A:[0,+\infty) \rightarrow$ $[0,+\infty)$ by $A(x)=\log (\psi(\exp x))$, for all $x \in[0,+\infty)$. Then $\mathrm{A}$ is continuous from right at all points such that $0<A(r)=$ $\log (\psi(\exp r))<r$ for $r>0$. Moreover,

$$
D(T x, T y) \leq \log \psi(\exp (\log d(x, y)))=A(D(x, y)), \text { for all } x, y \in X
$$

Then, the conditions of ([4], Theorem 3.7) are satisfied. So, by ([4], Theorem 3.7), the result follows.

Let us recall from [4] that a real valued function $\psi$ on $[a,+\infty)$ is upper semi continuous at $r \in[a,+\infty)$ from right, if $\limsup \psi\left(r_{j}\right) \leq \psi(r)$, whenever $r_{j} \rightarrow r$.

$j \rightarrow+\infty$

Theorem 4.9. Let $(X, d)$ be a complete EL metric space. Let a mapping $\psi:[1,+\infty) \rightarrow[1,+\infty)$ be upper semi continuous from right at all points such that $1<\psi(t)<t$ for $t>1$. Let $T:(X, d) \rightarrow(X, d)$ be a mapping such that $d(T x, T y) \leq \psi(d(x, y))$, for all $x, y \in X$. Then $T$ has a unique fixed point $x^{*}$. Also, for each $x_{0}$ in $X,\left(T^{n} x_{0}\right)_{n=1}^{+\infty}$ converges to $x^{*}$. 
Proof. Define $D$ and $A$ as in the proof of the previous theorem. Then $0<A(t)<t$ for $t>0$ and $A$ is upper semi continuous from right at all points. Moreover,

$$
D(T x, T y) \leq A(D(x, y)), \text { for all } x, y \in X,
$$

and $(X, D)$ is a complete metric space. Then, by ([4], Theorem 3.8), the result follows.

Theorem 4.10. Let $(X, d)$ be a complete EL metric space. Let $\psi:[1,+\infty) \rightarrow[1,+\infty)$ be a monotone non decreasing function such that $\lim _{n \rightarrow+\infty} \psi^{n}(t)=1$ for $t>1$. Let $T:(X, d) \rightarrow(X, d)$ be a mapping such that $d(T x, T y) \leq \psi(d(x, y))$, for all $x, y \in X$. Then $T$ has a unique fixed point $x^{*}$ and $\left(T^{n} x_{0}\right)_{n=1}^{+\infty}$ converges to $x^{*}$, for every $x_{0} \in X$.

Proof. Let $D=\log d$. Define $A:(0,+\infty) \rightarrow(0,+\infty)$ by $A(t)=\log (\psi(\exp t))$, for all $t \in(0,+\infty)$. Then $A$ is a monotone non decreasing function such that $A^{n}(t)=\log \left(\psi^{n}(\exp t)\right)$, for all $t>0$ and for all $n=1,2, \ldots$. So, $\lim _{n \rightarrow+\infty} A^{n}(t)=0$, for every $t>0$. Also,

$$
D(T x, T y) \leq A(D(x, y)), \text { for all } x, y \in X .
$$

Now the result follows from ([4], Theorem 3.10).

Notation 4.11. For a mapping $T:(X, d) \rightarrow(X, d)$ on an EL metric space $(X, d)$, let $k(T)=\sup \left\{\frac{\log d(T x, T y)}{\log d(x, y)}: x, y \in X, x \neq y\right\}$, and let $k_{+\infty}(T)=\limsup _{n \rightarrow+\infty}\left(k\left(T^{n}\right)\right)^{\frac{1}{n}}$.

Theorem 4.12. Let $(X, d)$ be a complete EL metric space. Let $T:(X, d) \rightarrow(X, d)$ be a continuous mapping for which $k_{+\infty}(T)<1$. Then $T$ has a unique fixed point $x^{*}$. Also, $\left(T^{n} x_{0}\right)_{n=1}^{+\infty}$ converges to $x^{*}$, for each $x_{0} \in X$.

Proof. Let $D=\log d$. Then the result follows from ([4], Theorem 3.11).

Theorem 4.13. Let $(X, d)$ be a complete EL metric space. Let $\phi: X \rightarrow[1,+\infty)$ be a mapping. Let $T:(X, d) \rightarrow(X, d)$ be a continuous mapping which satisfies $d(x, T x) \leq \frac{\phi(x)}{\phi(T x)}$, for all $x \in X$. Then $\left(T^{n} x_{0}\right)_{n=1}^{+\infty}$ converges to a fixed point of $T$, for each $x_{0} \in X$.

Proof. Let $D=\log d$. Define $A: X \rightarrow[0,+\infty)$ by $A(x)=\log (\phi(x))$, for all $x \in X$. Then $T:(X, D) \rightarrow(X, D)$ is a continuous mapping such that

$$
D(x, T x) \leq A(x)-A(T x), \text { for all } x \in X
$$

Now the result follows from ([4], Theorem 3.13).

Let us recall from [4] that a real valued function $\phi$ on a metric space $(X, D)$ is lower semi continuous at $x$, if $\phi(x) \leq r$, whenever $\lim _{n \rightarrow+\infty} x_{n}=x$ in $(X, D)$ and $\lim _{n \rightarrow+\infty} \phi\left(x_{n}\right)=r$ in the real line. In this case, it can also be stated that $\phi$ on $(X, d)$ is lower semi continuous at $x$, where $d=\exp D$, in view of Remark 2.7.

Theorem 4.14. Let $(X, d)$ be a complete EL metric space. Let $\phi:(X, d) \rightarrow[1,+\infty)$ be a function which is lower semi continuous at every point of $(X, d)$. Let $T: X \rightarrow X$ be a mapping which satisfies $d(x, T x) \leq \frac{\phi(x)}{\phi(T x)}$, for all $x \in X$. Then $T$ has a fixed point.

Proof. Let $D=\log d$. Define $A:(X, D) \rightarrow[0,+\infty)$ by $A(x)=\log (\phi(x))$, for all $x \in X$. Then $A$ is lower semi continuous at every point of $(X, d)$. Moreover,

$$
D(x, T x) \leq A(x)-A(T x), \text { for all } x \in X
$$

Now the result follows from ([4], Theorem 3.15).

Theorem 4.15. Let $\left(X, d_{X}\right)$ and $\left(Y, d_{Y}\right)$ be complete EL metric spaces. Let $T: X \rightarrow X$ be a mapping. Let $S:\left(X, d_{X}\right) \rightarrow\left(Y, d_{Y}\right)$ be a mapping having closed graph. Let $\phi:\left(S(X), d_{Y}\right) \rightarrow[1,+\infty)$ be a function which is lower semi continuous at every point of $\left(S(X), d_{Y}\right)$. Let $c>0$ be a constant such that

$$
\max \left\{d_{X}(x, T x),\left(d_{Y}(S x, S(T x))\right)^{c}\right\} \leq \frac{\phi(S x)}{\phi(S(T x))}, \text { for all } x \in X
$$

Then T has a fixed point. 
Proof. Let $D_{X}=\log d_{X}$ and $D_{Y}=\log d_{Y}$. Define $A:\left(S(X), D_{Y}\right) \rightarrow[0,+\infty)$ by $A(y)=\log \phi(y)$, for all $y \in S(X)$. Then $A$ is lower semi continuous at every point of $\left(S(X), D_{Y}\right)$. Also,

$$
\max \left\{D_{X}(x, T x), c D_{Y}(S x, S(T x))\right\} \leq A(S x)-A(S(T x)), \text { for all } x \in X .
$$

Now the result follows from ([4], Theorem 3.16).

Definition 4.16. Let $(X, d)$ be an EL metric space. Let $D=\log d$. Let $C B(X)$ denote the collection of all nonempty closed and bounded subsets of $(X, d)$ (or of $(X, D)$ ). For every $A, B \in C B(X)$, let

$$
P(A, B)=\max \left\{\sup _{y \in B} \inf _{x \in A} d(x, y), \sup _{y \in A} \inf _{x \in B} d(x, y)\right\}
$$

and let

$$
H(A, B)=\max \left\{\sup _{y \in B} \inf _{x \in A} D(x, y), \sup _{y \in A} \inf _{x \in B} D(x, y)\right\} .
$$

Then $H$ is the usual Hausdorff metric on $C B(X)$ derived from $D$. Then $P=\exp H$ on $C B(X)$ so that $P$ is an EL metric on $C B(X)$. Let us call the EL metric P as Pompieu EL metric.

Let us recall that if $(X, D)$ is a complete metric space, then $(C B(X), H)$ is also a complete metric space. The next theorem is a transform of the first fundamental fixed point theorem for set valued mappings.

Theorem 4.17. Let $(X, d)$ be a complete EL metric space. Let $P$ be the Pompeiu EL metric on CB $(X)$ derived from d. Let $k \in(0,1)$. Let $T: X \rightarrow C B(X)$ be a mapping such that $P(T x, T y) \leq(d(x, y))^{k}$, for all $x, y \in X$. Then there exists an element $x^{*} \in X$ such that $x^{*} \in T\left(x^{*}\right)$.

Proof. Let $D=\log d$ and $H=\log P$. Then $H(T x, T y) \leq k D(x, y)$, for all $x, y \in X$. Now, the result follows from ([4], Theorem 3.20).

Let $(X, d)$ be an EL metric space. For a subset $A$ of $X$, let us use the notation $\operatorname{diam} A$ for the $\operatorname{diameter} \sup \{d(x, y): x, y \in A\}$ in $(X, d)$. Let $D=\log d$. Let us use the notation Diam $A$ for the diameter $\sup \{D(x, y): x, y \in A\}$ in $(X, D)$. Let us observe that $\operatorname{Diam} A=\log \operatorname{diam} A$.

Theorem 4.18. Let $(X, d)$ be a bounded complete EL metric space. For every $x, y \in X$, let $O(x, y)=\left\{x, T x, T^{2}(x), \ldots, y, T y, T^{2} y, \ldots\right\}$. Let $\phi:[1,+\infty) \rightarrow[1,+\infty)$ be a non decreasing continuous function such that $\phi(s)<s$, for $s>1$. Let $T:(X, d) \rightarrow(X, d)$ be a continuous mapping. Suppose for each $x \in X$, there exists a positive integer $n(x)$ such that $d\left(T^{n} x, T^{n} y\right) \leq \phi($ diam $O(x, y))$, for all $y \in X$, for all $n \geq n(x)$. Then there exists a unique fixed point $x^{*}$ such that $\left(T^{n} x_{0}\right)_{n=1}^{+\infty}$ converges to $x^{*}$, for every $x_{0} \in X$.

Proof. Let $D=\log d$. Define $A:[0,+\infty) \rightarrow[0,+\infty)$ by $A(x)=\log (\phi(\exp x))$, for all $x \in[0,+\infty)$. Then $A$ is a non decreasing continuous function such that $A(s)<s$ for $s>0$. Also, for each $x \in X$ there exists a positive integer $n(x)$ such that

$$
D\left(T^{n} x, T^{n} y\right) \leq \log (\phi(\exp (\log (\operatorname{diam} O(x, y)))))=A(\operatorname{Diam} O(x, y)), \text { forally } \in X, \text { for all } n \geq n(x) .
$$

Here $T:(X, D) \rightarrow(X, D)$ is continuous. Now the result follows from ([4], Theorem 3.22).

The arguments used in the previous proof and ([4], Theorem 3.23) imply the following theorem.

Theorem 4.19. Let $(X, d), \phi$ and $O(x, y)$ be as described in the previous theorem. Let $T: X \rightarrow X$ be a mapping such that

$$
d(T x, T y) \leq \phi(\operatorname{diam} O(x, y)), \text { for all } x, y \in X .
$$

Then $T$ has a unique fixed point $x^{*}$. Also, for each $x_{0} \in X$, the sequence $\left(T^{n} x_{0}\right)_{n=1}^{+\infty}$ converges to $x^{*}$.

Let us next convert ([4], Exercise 3.3), which is ([12], Chapter 1, Theorem 5.1). Let us recall the notations used in Definition 2.4 and let us use the transformation $D=\log d$ for this purpose.

Theorem 4.20. Let $(X, d)$ be a complete EL metric space and Let $T: X \rightarrow X$ be a mapping. Assume that for each $\varepsilon>1$, there is a $\delta>1$ such that $T\left(B_{d}(x, \varepsilon)\right) \subseteq B_{d}(x, \varepsilon)$, whenever $d(x, T x)<\delta$. If $d\left(T^{n} x_{0}, T^{n+1} x_{0}\right) \rightarrow 1$, as $n \rightarrow+\infty$, for some $x_{0} \in X$, then the sequence $\left(T^{n} x_{0}\right)_{n=1}^{+\infty}$ converges to a fixed point $x^{*}$ of $T$ in $(X, d)$.

Let us again use the notations used in Definition 2.4 to transform ([4], Definition 4.1). 
Definition 4.21. Let $(X, d)$ be an EL metric space. Suppose, for any class $\left(C_{d}\left(x_{i}, r_{i}\right)\right)_{i \in I}$ of closed balls in $(X, d)$ which satisfy

$$
d\left(x_{i}, x_{j}\right) \leq r_{i} r_{j} \text { for all } i, j \in I,
$$

the intersection $\bigcap_{i \in I} C_{d}\left(x_{i}, r_{i}\right)$ is non empty. Then $(X, d)$ is said to be EL hyperconvex.

Remark 4.22. Suppose $(X, d)$ is EL hyperconvex. Let $D=\log d$. Then, for any class $\left(C_{D}\left(x_{i}, r_{i}\right)\right)_{i \in I}$ of closed balls in $(X, D)$ which satisfy

$$
D\left(x_{i}, x_{j}\right) \leq r_{i}+r_{j}, \text { for all } i, j \in I,
$$

the intersection $\bigcap_{i \in I} C_{D}\left(x_{i}, r_{i}\right)$ is non empty, because $C_{D}\left(x_{i}, r_{i}\right)=C_{d}\left(x_{i}, \exp r_{i}\right)$, for all $i \in I$, and

$$
d\left(x_{i}, x_{j}\right) \leq\left(\exp r_{i}\right)\left(\exp r_{j}\right), \text { for all } i, j \in I
$$

Then, by ([4], Definition 4.1), the metric space $(X, D)$ is hyperconvex. On the other hand, if $(X, D)$ is a hyperconvex metric space, then the EL metric space $(X, d)$ is EL hyperconvex, where $d=\exp D$.

Example 4.23. Consider the vector space $l^{\infty}$ of all bounded real sequences with the usual metric D defined by

$$
D\left(\left(x_{n}\right)_{n=1}^{+\infty},\left(y_{n}\right)_{n=1}^{+\infty}\right)=\sup _{n=1,2, \ldots}\left|x_{n}-y_{n}\right| .
$$

Then by ([4], Proposition 4.2) and ([4], Theorem 4.2), $\left(l^{\infty}, D\right)$ is hyperconvex and hence $\left(l^{\infty}, d\right)$ is EL hyperconvex, where $d=\exp D$.

Theorem 4.24. Let $(X, d)$ be a bounded EL hyper convex EL metric space. Let $T: X \rightarrow X$ be a mapping such that $d(T x, T y) \leq d(x, y)$, for all $x, y \in X$. Then the fixed point set $\{x \in X: T x=x\}$ is non empty and it is EL hyper convex with respect to $d$.

Proof. Let $D=\log d$. Then $D(T x, T y) \leq D(x, y)$, for all $x, y \in X$. Now, the result follows from ([4], Theorem 4.8).

Definition 4.25. Let $(X, d)$ be an EL metric space, and let $D=\log d$. Let $A$ be a subset of $X$. Let $\operatorname{cov}_{d}(A)\left(\operatorname{or}_{\text {, cov }}(A)\right)$ denote the intersection of all closed balls in $(X, d)\left(\right.$ or, in $(X, D)$, respectively) which contain $A$. Since $C_{D}(x, \log r)=C_{d}(x, r)$, for $r \geq 1$ and $x \in X$, then $\operatorname{cov}_{d}(A)=\operatorname{cov}_{D}(A)$, for any subset $A$ of $X$.

A bounded set $A$ in a metric space $(X, D)$ is an admissible subset of $X$, according to ([4], Definition 4.2), if $A=\operatorname{cov}_{D}(A)$. So, let us say that a bounded subset $A$ of an $\operatorname{EL}$ metric space $(X, d)$ is admissible when $A=\operatorname{cov}_{d}(A)$, because $\operatorname{cov}_{d}(A)=\operatorname{cov}_{D}(A)$ for $D=\log d$. Following the book [4], let us write $A(X)$ for the collection of all admissible subsets of $(X, d)$ or $(X, D)$.

The next definition is ([4], Definition 5.1) for metric spaces.

Definition 4.26. Let $(X, D)$ be a metric space. Then $A(X)$ is said to be compact, if every descending chain non empty members of $A(X)$ has non empty intersection. Let us follow the same terminology even for $(X, d)$, where $d=\log D$.

Let $(X, D)$ be a metric space and let $d=\exp D$. Let $A$ be a subset of $X$. Let $r_{D}(A)=\inf \{\sup \{D(x, y): y \in A\}: x \in A\}$ and $r_{d}(A)=\inf \{\sup \{d(x, y): y \in A\}: x \in A\}$. Then $r_{d}(A)=\exp r_{D}(A)$. Hence $r_{d}(A)<\operatorname{diam} A$ if and only if $r_{D}(A)<D i a m A$.

The next definition is ([4], Definition 5.2) given for metric spaces.

Definition 4.27. Let $(X, D)$ be a metric space. Then $A(X)$ is said to be normal if $r_{D}(A)<$ Diam $A$, whenever $A \in A(X)$ and Diam $A>0$. Equivalently, $A(X)$ is said to be normal if $r_{d}(A)<\operatorname{diam} A$, whenever $A \in A(X)$ and $\operatorname{diam} A>1$, where $d=\log D$.

The following is an obvious transformed form of ([4], Theorem 5.1).

Theorem 4.28. Let $(X, d)$ be a non empty bounded EL metric space for which $A(X)$ is compact and normal. Let $T: X \rightarrow X$ be a mapping such that $d(T x, T y) \leq d(x, y)$, for all $x, y \in X$. Then $T$ has atleast one fixed point.

From the results which have been derived, it can be observed that a transformation for a fixed point result requires transformations for some concepts. It has been established indirectly that there is an assurance for transformations of metric fixed point results to EL metric fixed point results. Only one thing should be observed: Results to be transformed should depend only on metrics. For example, let us consider ([4], Corollary 8.4) which states that if $T: A \rightarrow A$ is a mapping on an admissible subset $A$ of $\left(l_{1},\|\|_{1}\right)$ such that $\|T x-T y\|_{1} \leq\|x-y\|_{1}$, for all $x, y \in A$, then $T$ has a fixed point in $A$. It seems to be a result 
for a normed space depending on algebraic structures; but it is a result for a metric space which is independent of algebraic structures. So, it is a result for the EL metric space $\left(l_{1}, d\right)$ when

$$
d\left(\left(x_{n}\right)_{n=1}^{+\infty},\left(y_{n}\right)_{n=1}^{+\infty}\right)=\prod_{n=1}^{+\infty} e^{\left|x_{n}-y_{n}\right|}, \text { for all }\left(x_{n}\right)_{n=1}^{+\infty},\left(y_{n}\right)_{n=1}^{+\infty} \in l_{1} .
$$

For this metric $d$, ([4], Corollary 8.4) can be transformed to the following form.

Theorem 4.29. Let $A$ be an admissible subset of $\left(l_{1}, d\right)$. Let $T: A \rightarrow A$ be a mapping such that $d(T x, T y) \leq d(x, y)$, for all $x, y \in X$. Then $T$ has a fixed point in A.

A continuation is separated by the following conclusion of this section.

Conclusion 4.30. Convert hypotheses of a metric fixed point result by means of exponential transform to get hypotheses for an EL metric fixed point result. For a proof of the EL metric fixed point result, use logarithmic transform.

\section{More transformed results}

The next definition is proposed for transformed norms.

Definition 5.1. Let $X$ be a vector space over the field of real numbers or the field of complex numbers. Let $\|\mid\|$ be a real valued function on $X$ satisfying the following conditions, where $\|x\|$ is \|\|$(x)$ :

(i) $\|x\| \geq 1$, for all $x \in X$, and $\|x\|=1$ if and only if $x=0$ in $X$.

(ii) $\|\lambda x\|=\|x\|^{|\lambda|}$, for all $x \in X$, and for all scalars $\lambda$.

(iii) $\|x+y\| \leq\|x\|\|y\|$, for all $x, y \in X$.

Then, let us call || || as EL norm, and (X, || ||) as EL normed space.

Example 5.2. If $(X,\|\|)$ is a normed space, then \|\|$_{0}$ is defined by $\|x\|_{0}=8^{\pi\|x\|}$, for all $x \in X$, is an EL norm on $X$. On the other hand, if $(X,\|\|)$ is an EL normed space, then $\|x\|_{0}$ defined by $\|x\|_{0}=\log \|x\|$, for all $x \in X$, is a norm on $X$.

Let us discuss fixed point theorems again on metric spaces. Let us first transform the statement of ([4], Theorem 3.4), but let us present a direct proof (out line).

Corollary 5.3. Let $X$ be a non empty set with two EL metrics $d_{1}$ and $d_{2}$. Suppose $d_{1}(x, y) \leq d_{2}(x, y)$, for all $x, y \in X$. Suppose $\left(X, d_{1}\right)$ be complete. Let $T:\left(X, d_{1}\right) \rightarrow\left(X, d_{1}\right)$ be a continuous mapping such that $d_{2}(T x, T y) \leq\left(d_{2}(x, y)\right)^{k}$, for all $x, y \in X$, for some $k \in(0,1)$. Then $T$ has a unique fixed point $x^{*}$.

Proof. Fix $x_{0} \in X$. Then $\left(T^{n} x_{0}\right)_{n=1}^{+\infty}$ is a Cauchy sequence in $\left(X, d_{2}\right)$, because $d_{2}(T x, T y) \leq\left(d_{2}(x, y)\right)^{k}$, for all $x, y \in X$. Since $d_{1}(x, y) \leq d_{2}(x, y)$, for all $x, y \in X$, the sequence $\left(T^{n} x_{0}\right)_{n=1}^{+\infty}$ is Cauchy also in $\left(X, d_{1}\right)$. Let $\left(T^{n} x_{0}\right)_{n=1}^{+\infty}$ converge to $x^{*}$ in $\left(X, d_{1}\right)$. Then $x^{*}$ is a fixed point of $T$, because $T:\left(X, d_{1}\right) \rightarrow\left(X, d_{1}\right)$ is continuous. The uniqueness of $x^{*}$ follows, because $k \in(0,1)$.

One can introduce $D_{1}=\log d_{1}, D_{2}=\log d_{2}$ and apply ([4], Theorem 3.4) to obtain the previous Theorem 5.3. But a direct proof has been presented just to observe that there is a technique of sharing conditions in hypotheses in a metric fixed point result between two metrics. This sharing technique is also a simple technique for converting results for generalizations. This sharing technique is also indirectly applied by using metric preserving functions; see [13]. For a good introduction about results on metric preserving functions, one may see the article [14].

Definition 5.4. Let us say that a function $f:[1,+\infty) \rightarrow[1,+\infty)$ is EL metric preserving, if $f \circ d$ is an EL metric for every EL metric $d$. It is said to be strongly metric preserving, if the topologies induced by $d$ and $f \circ d$ coincide, for every EL metric $d$.

The exercise problem ([11], Chapter 4, Problem $C$ ) is converted to the following result.

Proposition 5.5. Let $f:[1,+\infty) \rightarrow[1,+\infty)$ be a non decreasing continuous function such that $f(x)=1$ if and only if $x=1$ and such that $f(x y) \leq f(x) f(y)$, for all $x, y \in[1,+\infty)$. Then $f$ is a strongly EL metric preserving function.

Proof. Define $F:[0,+\infty) \rightarrow[0,+\infty)$ by $F(x)=\log (f(\exp x))$. Then $F$ is continuous, non decreasing, and $F(x)=0$ if and only if $x=0$ in $[0,+\infty)$. Moreover,

$$
F(x+y)=\log (f(\exp (x+y))) \leq \log (f(\exp x))+\log (f(\exp y))=F(x)+F(y), \text { for all } x, y \in[0,+\infty) .
$$

Then $F$ is a strongly metric preserving function (see [14], for definition). Then $F \circ \log d$ is a metric whenever $d$ is an EL metric, and the metrics $\log d, F \circ \log d$ induce a common topology. Hence,

$$
\exp \circ F \circ \log d=\exp \circ \log \circ f \circ \exp \circ \log d=f \circ d
$$

is an EL metric whenever $d$ is an EL metric, and the EL metrics $f \circ d$ and $d$ induce the same topology. 
Let us now state a simplified version of ([4], Theorem 3.17) in transformed form.

Theorem 5.6. Let $(X, d)$ be a complete EL metric space. Let $r>0$. Let $\phi:(X, d) \rightarrow[r,+\infty)$ be a function. Let $\psi:[1,+\infty) \rightarrow$ $[1,+\infty)$ be an EL metric preserving function, which is strictly increasing. Let $T:(X, d) \rightarrow(X, d)$ be a continuous mapping which satisfies $\psi(d(x, T x)) \leq \frac{\phi(x)}{\phi(T x)}$, for all $x \in X$. Then $T$ has a fixed point.

Proof. Let $d_{1}=\psi \circ d$. Fix $x_{0} \in X$. Let $x_{n}=T^{n} x_{0}$, for all $n=1,2, \ldots$. Then

$$
1 \leq d_{1}\left(x_{n}, x_{n+1}\right) \leq \frac{\phi\left(x_{n}\right)}{\phi\left(x_{n+1}\right)}, \text { for all } n=1,2, \ldots
$$

For $n>m$,

$$
1 \leq d_{1}\left(x_{m}, x_{n}\right) \leq d_{1}\left(x_{m}, x_{m+1}\right) d_{1}\left(x_{m+1}, x_{m+2}\right) \ldots d_{1}\left(x_{n-1}, x_{n}\right) \leq \frac{\phi\left(x_{m}\right)}{\phi\left(x_{n}\right)} .
$$

So, $\left(\phi\left(x_{n}\right)\right)_{n=1}^{+\infty}$ is a monotone non increasing sequence. Let $\phi\left(x_{n}\right) \rightarrow M \geq r>0$, as $n \rightarrow+\infty$. Then, by the previous inequality, $d_{1}\left(x_{m}, x_{n}\right) \rightarrow 1$ as $n, m \rightarrow+\infty$. That is, $(\psi \circ d)\left(x_{m}, x_{n}\right) \rightarrow 1$ as $n, m \rightarrow+\infty$. Therefore, $d\left(x_{m}, x_{n}\right) \rightarrow 1$ as $n, m \rightarrow+\infty$, because $\psi$ is strictly increasing. Since $(X, d)$ is complete, $\left(x_{n}\right)_{n=1}^{+\infty}$ converges to some point $x^{*}$ in $(X, d)$. Since $T:(X, d) \rightarrow(X, d)$ is continuous, and $\left(T x_{n}\right)_{n=1}^{+\infty}$ converges to $x^{*}$, then $T x^{*}=x^{*}$.

Corollary 5.7. Let $(X, D)$ be a complete metric space. Let $\phi$ be a bounded below real valued function on (X,D). Let $\psi:[0,+\infty) \rightarrow[0,+\infty)$ be a strictly increasing metric preserving function. Let $T:(X, D) \rightarrow(X, D)$ be a continuous mapping which satisfies the condition $\psi(D(x, T x)) \leq \phi(x)-\phi(T x)$, for all $x \in X$. Then $T$ has a fixed point.

Proof. Let $d=\exp D$. Define a real valued function $A$ on $(X, d)$ by $A(x)=\exp (\phi(x))$, for all $x \in X$. Then, there is a number $r>0$ such that $A(x) \geq r$, for all $x \in X$. Define $B:[1,+\infty) \rightarrow[1,+\infty)$ by $B(s)=\exp (\psi(\log s))$, for all $s \geq 1$. Then B is a strictly increasing EL metric preserving function. Moreover,

$$
\begin{aligned}
B(d(x, T x))=\exp (\psi(\log d(x, T x)))=\exp (\psi(D(x, T x))) & \leq \exp (\phi(x)-\phi(T x)) \\
& =\frac{A(x)}{A(T x)}, \text { for all } x \in X
\end{aligned}
$$

Also, $T:(X, d) \rightarrow(X, d)$ is continuous, and $(X, d)$ is complete. Now, the result follows from the previous theorem.

Remark 5.8. The function $\psi$ given in Theorem 5.6 should be continuous at 1, and the function $\psi$ given in Corollary 5.7 should be continuous at 0. So, by ([14], Theorem 3.4), $\psi$ should be a strongly EL metric preserving function in Theorem 5.6 and $\psi$ should be a strongly metric preserving function in Corollary 5.7. In both results, the conditions on $\psi$ may be replaced by following simple conditions. For Theorem 5.6, assume that $\psi$ is a strongly EL metric preserving function. For Corollary 5.7, assume that $\psi$ is a strongly EL metric preserving function.

Conclusion 5.9. There are standard methods in literature to convert results for extensions and generalizations. ExponentialLogarithmic transformation method can be extended to derive many transformed results in analysis.

\section{Applications}

It has been mentioned in Section 4 that the main advantage of transformed statements lies in verifying conditions in examples. Let us modify slightly Example 4.1 for justification of this sentence.

Example 6.1. Let $X=\left[\frac{1}{2},+\infty\right]$. Let $d(x, y)=\max \left\{x y^{-1}, y x^{-1}\right\}$, for all $x, y \in X$. Let $D(x, y)=|x-y|$, for all $x, y \in X$. Then $(X, d)$ is a complete EL metric space and $(X, D)$ is a complete metric space. Let $T: X \rightarrow X$ be defined by $T x=x^{\frac{1}{2}}$, for all $x \in X$. Then,

$$
D(x, y)=|x-y|=\left|x^{\frac{1}{2}}-y^{\frac{1}{2}}\right|\left|x^{\frac{1}{2}}+y^{\frac{1}{2}}\right|=D(T x, T y)\left|x^{\frac{1}{2}}+y^{\frac{1}{2}}\right|, \text { for all } x, y \in X .
$$

Thus, there is no constant $M>0$ such that

$$
D(T x, T y) \leq M D(x, y), \text { for all } x, y \in X .
$$

Hence, the Banach contraction principle is not applicable in this case. However,

$$
1 \leq d(T x, T y)=\max \left\{\left(\frac{x}{y}\right)^{\frac{1}{2}},\left(\frac{y}{x}\right)^{\frac{1}{2}}\right\}=(d(x, y))^{\frac{1}{2}} \leq(d(x, y))^{k}, k \in\left[\frac{1}{2}, 1\right) \text {, for all } x, y \in X .
$$

By Theorem 3.3, for $x_{0} \in X$, the sequence $\left(T^{n} x_{0}\right)_{n=1}^{+\infty}$ converges to the unique fixed point 1 . 
Example 6.2. Let $X, d, D$ be as in the previous Example 6.1. Let $T: X \rightarrow X$ be defined by $T x=\frac{x+1}{2}$, for all $x \in X$. Then

$$
d(T x, T y)=\max \left\{\frac{x+1}{y+1}, \frac{y+1}{x+1}\right\}
$$

and

$$
d(x, y)=\max \left\{\frac{x}{y}, \frac{y}{x}\right\}
$$

Hence, there is no $k \in(0,1]$ such that

$$
d(T x, T y) \leq(d(x, y))^{k}, \text { for all } x, y \in X
$$

Thus, Theorem 3.3 is not applicable in this case. However,

$$
D(T x, T y)=\frac{1}{2}|x-y|=\frac{1}{2} D(x, y), \text { for all } x, y \in X .
$$

Then, by the Banach contraction principle, for each $x_{0} \in X$, the sequence $\left(T^{n} x_{0}\right)_{n=1}^{+\infty}$ converges to the unique fixed point 1 .

Conclusion 6.3. Results in both metric fixed point theory and EL metric fixed point theory are applicable, and both of them should be developed.

\section{Final conclusions}

Both metric fixed point theory and EL metric fixed point theory should be developed, as it has been explained by Example 6.1 and Example 6.2. Some more things have to be concluded for transformations.

The open mapping theorem and the closed graph theorem are equivalent in the sense that if one theorem is assumed then the other theorem can be derived from the first one. One may apply these theorems according to convenience in any particular format. A similar event happens in measure theory. Lebesgue monotone convergence theorem, Fatou's lemma and Lebesgue dominated convergence theorem are equivalent. They are applied according to convenience. So, there is a need to transform arguments and results of fixed point theory, because of the same reason.

The transformations used in this article are natural ones, but not trivial ones. The ultimate aim of transformation is to increase possibilities to derive new examples and results. The most expected application of fixed point theory is to increase possibilities to solve equations. The transform used in this article can be extended to many branches of analysis.

\section{Acknowledgement}

Dr. C. Ganesa Moorthy (Professor, Department of Mathematics, Alagappa University, Karaikudi- 630003, INDIA) gratefully acknowledges the joint financial support of RUSA-Phase 2.0 grant sanctioned vide letter No.F 24-51/2014-U, Policy (TN Multi-Gen), Dept. of Edn. Govt. of India, Dt. 09.10.2018, UGC-SAP (DRS-I) vide letter No.F.510/8/DRS-I/2016 (SAP-I) Dt. 23.08.2016 and DST (FIST - level I) 657876570 vide letter No.SR/FIST/MS-I/2018-17 Dt. 20.12.2018.

\section{References}

[1] T. V. An, N. V. Dung, Z. Kadelburg, S. Radenovic, Various generalizations of metric spaces and fixed point theorems, Rev. R. Acad. Cienc. Exactas Fis. Nat. Ser. A., 109 (2015), 175-198.

[2] T. Dosenovic, M. Postolache, S. Radenovic, On multiplicative metric spaces: Survey, Fixed Point Theory Appl., 2016 (2016), Article ID 92, 17 pages, doi: 10.1186/s13663-016-0584-6.

[3] A. E. Bashirov, E. M. Kurpinar, A. Özyapici, Multiplicative calculus and its applications, J. Math. Anal. Appl., 337(1) (2008), $36-48$.

[4] M. K. Khamsi, W. A. Kirk, An Introduction to Metric Spaces and Fixed Point Theory, John Wiley, New York, 2001.

[5] C.G. Moorthy, S.I. Raj, Inverse fixed points of sequences of mappings, Asian-Eur. J. Math., 2020(2020), Article ID 2150027, 8 pages, doi: $10.1142 / \mathrm{S} 1793557121500273$.

[6] M. Candan, Domain of the double sequential band matrix in the classical sequence spaces, J. Inequal. Appl., 2012(2012), Article ID 281, 15 pages, doi.org/10.1186/1029-242X-2012-281.

[7] M. Candan, A new sequence space isomorphic to the space l( $p$ ) and compact operators, J. Math. Comput. Sci., 4(2)(2014), 306-334.

[8] M. Candan, Some new sequence spaces derived from the spaces of bounded, convergent and null sequences, Int. J. Mod. Math. Sci., 12(2)(2014),

[9] M. Candan, Domain of the double sequential band matrix in the spaces of convergent and null sequences, Adv. Differ. Equ. 2014 (2014), Article ID 163, 18 pages, doi: 10.1186/1687-1847-2014-163.

[10] M.Ozavsar, A.C. Cevikel, Fixed points of multiplicative contraction mappings on multiplicative metric spaces, (2012), arXiv:1205.5131v1 [math.GM].

[11] J. L. Kelly, General Topology, Von Nostrand, London, 1955.

[12] A. Granas, J. Dugundji, Fixed Point Theory, Springer, New York, 2003.

[13] P. Pongsriiam, I. Termwuttipong, On metric preserving functions and fixed point theorems, Fixed Point Theory Appl., 2014 (2014), Article ID 179, 14 pages, doi: 10.1186/1687-1812-2014-179.

[14] P. Corazza, Introduction to metric preserving functions, Amer. Math. Monthly, 104 (1999), 309-323. 